\title{
An Interesting Case of Double Compound Intussusception without Intestinal Occlusion in a 5-Year-Old Boy
}

\author{
Francesca Destro ${ }^{1}$ Noemi Cantone ${ }^{1}$ Michela Maffi $^{1}$ Tommaso Gargano ${ }^{1}$ Mario Lima ${ }^{1}$ \\ ${ }^{1}$ Department of Pediatric Surgery, S. Orsola-Malpighi Hospital, \\ Bologna, Italy \\ Address for correspondence Mario Lima, PhD, Department of \\ Pediatric Surgery, S. Orsola Malpighi Hospital, Via Massarenti 11, \\ Bologna 40138, Italy (e-mail: mario.lima@unibo.it). \\ Eur J Pediatr Surg Rep 2014;2:20-22.
}

\begin{abstract}
Keywords

- compound intussusception

- children

- laparoscopy

Intussusception is a very common surgical finding in children. Idiopathic intussusception is relatively frequent in children within the first year and it usually causes an acute abdomen. Instead, complex intussusception involving multiple or remote intestinal segments is a very rare entity and it is frequently diagnosed intraoperatively. It is often because of an anatomical cause and it can occur at any age. We report a unique case of contemporary double site anterograde and retrograde ileoileal intussusception without intestinal occlusion because of a submucous intestinal lipoma. In our case, imaging studies were important for clinical suspicion and laparoscopy was essential for final diagnosis and its resolution.
\end{abstract}

\section{Introduction}

Intussusception is a common cause of bowel obstruction in children. Beside the classical presentation, there are complex cases involving multiple adjacent or remote segments. We describe an unusual case of double compounded ileoileal intussusceptions because of a submucous intestinal lipoma. Laparoscopically assisted resolution of the intussusceptions was successfully accomplished.

\section{Case Report}

A previously well 5-year-old boy with a 7-day history of crampy abdominal pain was admitted at our hospital for presumed abdominal mass. The child's mother denied any other symptom except for abdominal pain and she gave history of constipation on and off for the last month. Stool was never suspicious for blood. The abdominal mass was pointed out on ultrasound and computed tomography (CT) scans performed at another hospital. Radiological investigations showed an inhomogeneous mass with vessels and adipose tissue in it. The mass was $4.6-\times 4.7-\times 10-\mathrm{cm}$ long and it was located in the left part of the abdomen. No signs of intestinal occlusion were identified. On admission at our department, the physical examination showed a soft, non tender mass just above the umbilicus, extended along the descendent colon. The rest of systemic examination was unremarkable. Laboratory tests were normal. The plain abdominal radiograph showed non air-fluid levels nor free air in the abdomen. Sonographic examination raised the suspicion of intussusceptions with intestinal wall thickening and hyperecoic. We decided to perform further investigations since the patient was asymptomatic: tumoral markers were normal and retrograde enema showed regular canalization of the colon and of the last ileal tract. We had time to discuss the case with radiologists and to revise CT scans. Images showed a mass composed of multiple concentric circles (triple circle mass) ( - Fig. 1 a,b). A laparoscopy was performed with one $5 \mathrm{~mm}$ optic (umbilicus) and two operative trocars (flanks). A long intussuscepted ileal tract was identified ( - Fig. 2 a,b). The attempt to reduce the intussusceptions failed and the bowel was extracted after a lower enlargement of the umbilical wound. After manual reduction of the first retrograde segment, we noted a second anterograde intussuscepted received

September 19, 2013

accepted

October 19, 2013

published online

February 10, 2014

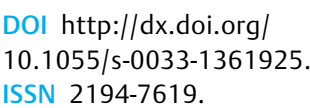

DOI http://dx.doi.org/

10.1055/s-0033-1361925.

ISSN 2194-7619.

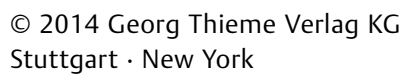

License terms

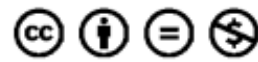




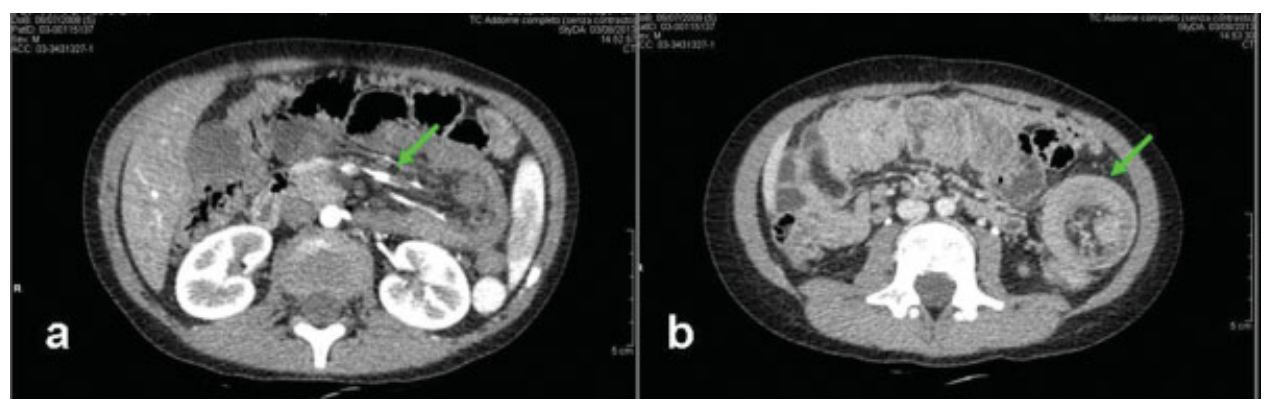

Fig. 1 Computed tomographic scans. (a) A long mass with vessels in it is visible. (b) In transversal section multiple circles appear.

segment proximal to the first one. These two segments made a compound intussusception. This part was reduced too. When the reduction was completed, the bowel appeared well perfused and it had a palpable, intraluminal mass. We performed a $7 \mathrm{~cm}$ long resection of the tract containing the mass (-Fig. 3 a,b,c,d). Histological examination showed a submucous intestinal lipoma. Recanalization was obtained with end to end anastomosis. We also explored the entire bowel identifying another ileoileal compound intussusceptions that was reduced. At the end of the procedure, a complementary appendectomy was performed. There were no postoperative complications and the boy was discharged 7 days after surgery.

\section{Discussion}

Intussusception is one of the most frequent cause of bowel obstruction in children younger than two years. Ileoileal intussusception is rare and it is often due to a lead point in older patients ${ }^{1}$. Complex intussusception is even rarer and it is diagnosed during surgical intervention. ${ }^{2}$ In classical cases proximal intestinal segment prolapses into the adjacent distal one. The displacement of more than one segment gives rise to complex entities. There is not a specific terminology regarding these forms that are described by various and interchangeable definitions. As described ${ }^{3}$ compound intussusception is a combination of anterograde and retrograde intussusception or double anterograde. ${ }^{4}$ This entity differs from the multiple (double or more) intussusception that involves separate points of the bowel. ${ }^{4,5}$ Multiple intussusception are often associated with congenital or acquired lead points that may cause abnormal motility. ${ }^{3,6}$ Both the small bowel and the colon may be involved. Intussusception can lead to ischemic intestinal alterations determining an acute abdomen. However symptoms can be blurred and the classical triad (palpable abdominal mass, abdominal pain and red currant jelly stool) is not always present. ${ }^{7}$ In our case a double and a compound intussusception were contemporary present but they did not cause bowel's alterations, nor acute symptoms. Radiological evaluations led us to think about intussusception. In particular the triple-circle appearance is pathognomonic (the outer circle is the distal segment, the middle is the second prolapsed segment and the third is the first one). ${ }^{2}$ Laparoscopy was required as radiological procedures failed to identify and reduce the intussusception. We usually achieve intussusception reduction with an hydrostatic or contrast enema but this procedure is scarcely effective when there is a lead point or an high part of the small bowel is involved. Moreover enemas can't be decisive in these cases and they may be dangerous in case of retrograde segments. We routinely perform laparoscopic exploration that allows the identification of the site of intussusception and its gentle reduction. If necessary involved bowel can be extracted through the umbilical wound. We suggest to always perform a careful evaluation of the bowel to identify multiple distant segments that may be involved, as happened in our case.

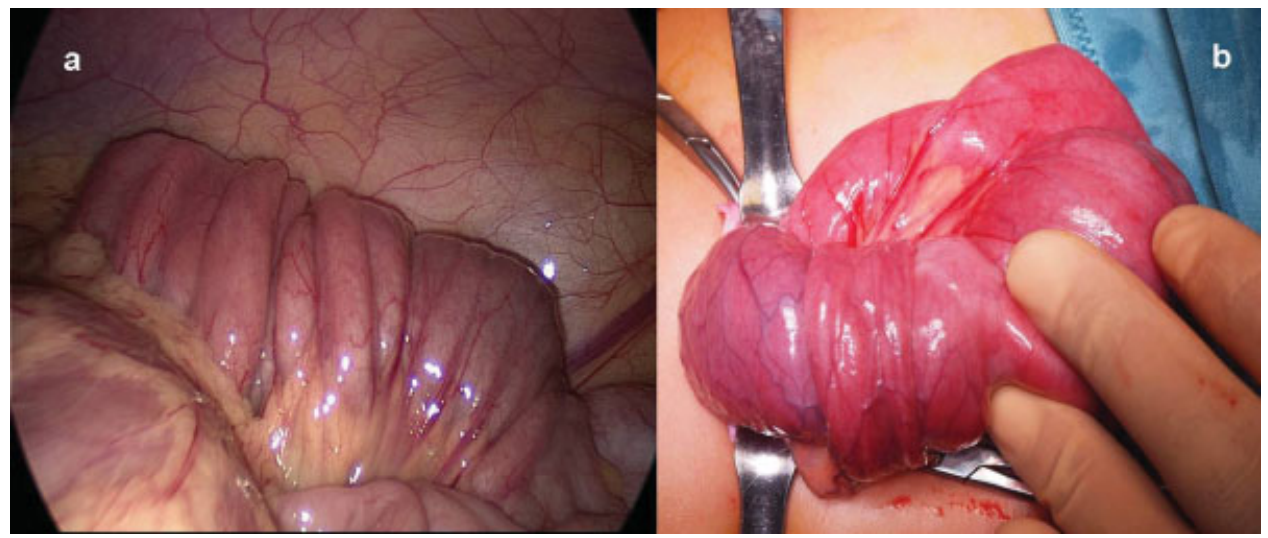

Fig. 2 (a) Laparoscopic vision of the intussuscepted ileal tract and (b) its appearance after exteriorization trough the enlarged umbilical wound. 


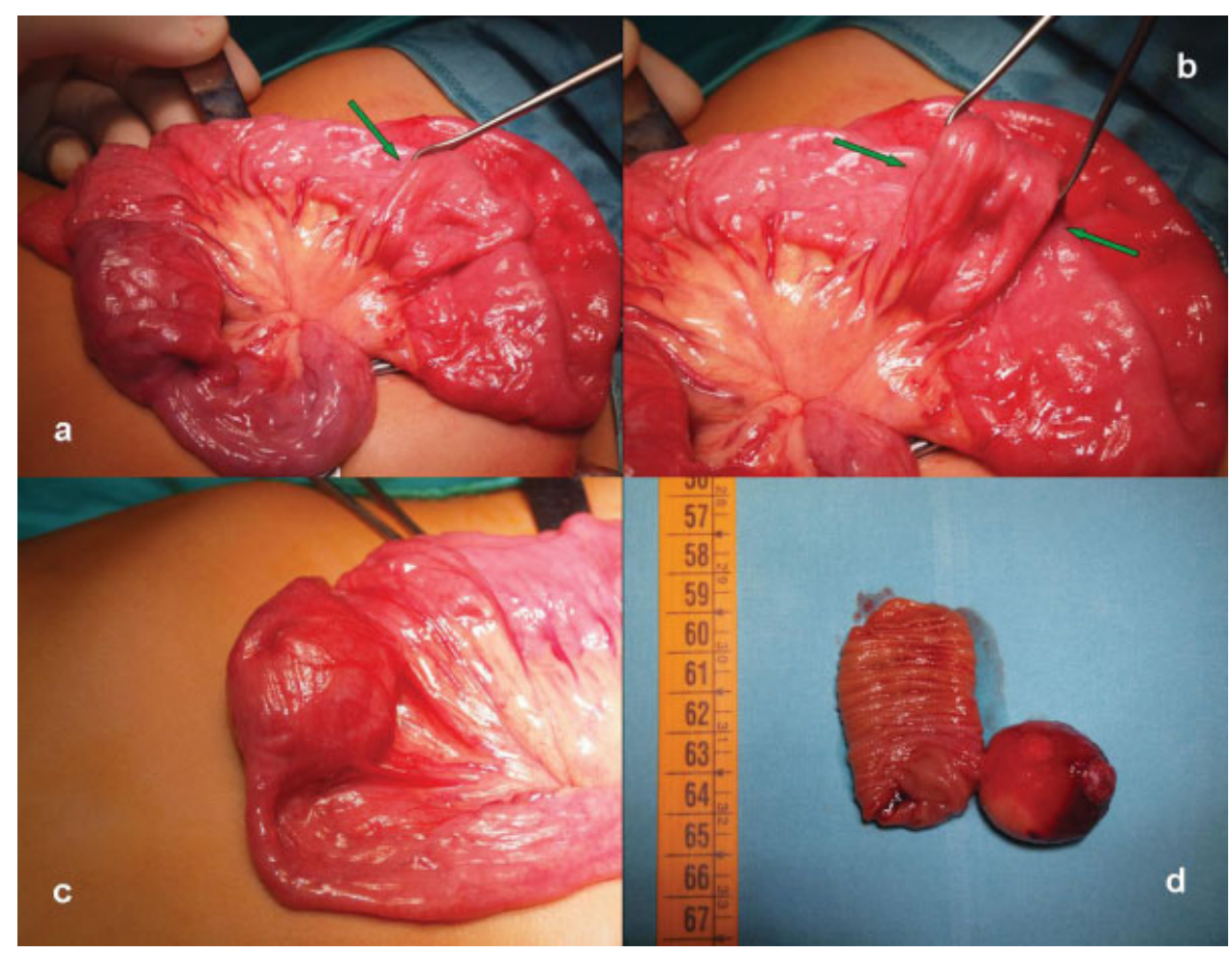

Fig. 3 (a, b) After the reduction of the first compound intussusception, another compound intussusception was noted (c) far from the mass. (d) The resected tract showed an intraluminal lipoma.

\section{Conclusions}

Intussusception is a common cause of intestinal occlusion in infants but it may present also in older children without acute abdomen. Laparoscopy is a safe and feasible technique and it can be used for diagnosis and therapy. We recommend to explore all the bowel to exclude the presence of complex forms.

\section{References}

1 Seifarth FG, Ryan ML, Triana J, et al. Diagnosis and laparoscopic treatment of ileoileal intussusception secondary to heterotopic pancreas in an infant: case report and review of the literature. J Pediatr Surg 2011;46(2):e33-e36
2 Kazez A, Ozel SK, Kocakoc E, Kiris A. Double intussusception in a child: the triple-circle sign. J Ultrasound Med 2004;23(12): 1659-1661

3 Arnold M, Sidler D, Moore SW. Compound colonic intussusception: a reason for failure of pneumatic reduction. J Pediatr Surg 2010;45(10):e25-e28

4 Kiyan G, Tugtepe H, Iskit SH, Dagli TE. Double intussusception in an infant. J Pediatr Surg 2002;37(11):1643-1644

5 Him FP, Weng YK, Hoi CW. A case of double compound intussusception in an infant. Singapore Med J 1980;21(2): 540-541

6 Scholz S, Loff S, Wirth H. Double ileoileal intussusception caused by a giant polypoid mass of heterotopic pancreas in a child. Eur J Pediatr 2000;159(11):861-862

7 Shiu JR, Chao HC, Chen CC, Chi CY. Rare concurrent ileoileal and ileocolic intussusceptions in a child presenting with painless hematochezia. Pediatr Neonatol 2010;51(6):359-362 